\title{
The price of risk and ambiguity in an intertemporal general equilibrium model of asset prices
}

\author{
Gonçalo Faria • João Correia-da-Silva
}

Received: 23 December 2011 / Accepted: 17 March 2012 / Published online: 25 April 2012

(C) Springer-Verlag 2012

\begin{abstract}
We consider a version of the intertemporal general equilibrium model of Cox et al. (Econometrica 53:363-384, 1985) with a single production process and two correlated state variables. It is assumed that only one of them, $Y_{2}$, has shocks correlated with those of the economy's output rate and, simultaneously, that the representative agent is ambiguous about its stochastic process. This implies that changes in $Y_{2}$ should be hedged and its uncertainty priced, with this price containing risk and ambiguity components. Ambiguity impacts asset pricing through two channels: the price of uncertainty associated with the ambiguous state variable, $Y_{2}$, and the interest rate. With ambiguity, the equilibrium price of uncertainty associated with $Y_{2}$ and the equilibrium interest rate can increase or decrease, depending on: (i) the correlations between the shocks in $Y_{2}$ and those in the output rate and in the other state variable; (ii) the diffusion functions of the stochastic processes for $Y_{2}$ and for the output rate; and (iii) the gradient of the value function with respect to $Y_{2}$. As applications of our generic setting, we deduct the model of Longstaff and Schwartz (J Financ 47:1259$1282,1992)$ for interest-rate-sensitive contingent claim pricing and the variance-risk price specification in the option pricing model of Heston (Rev Financ Stud 6:327343,1993 ). Additionally, it is obtained a variance-uncertainty price specification that can be used to obtain a closed-form solution for option pricing with ambiguity about stochastic variance.
\end{abstract}

G. Faria $(\varangle)$

Facultade de Ciencias Económicas e Empresariais, RGEA,

Universidad de Vigo, Lagoas Marcosende, 36310 Vigo, Spain

e-mail: gfaria@uvigo.es

J. Correia-da-Silva

CEF.UP and Faculdade de Economia, Universidade do Porto,

Rua Dr. Roberto Frias, 4200-464 Porto, Portugal

e-mail: joao@fep.up.pt 
Keywords Ambiguity - Asset pricing - Equilibrium price of uncertainty $\cdot$ Robust optimization

\section{JEL Classification $\quad$ C68 $\cdot$ D $81 \cdot$ G13}

\section{Introduction}

There are two major approaches for the modeling of asset prices and of the implied uncertainty prices: the equilibrium and the arbitrage approach.

The equilibrium approach includes models that start by describing the production sector of the economy, which is typically a set of production processes driven by exogenous state variables whose dynamics are, in turn, described by stochastic processes. The assets are contingent claims to the output of these production processes. With the objective of maximizing an utility function, the representative agent decides how much to consume and how much to invest (either physically in the production processes or financially by acquiring assets). The equilibrium prices of the assets, and the corresponding uncertainty prices, must be such that demand equals supply. An example of this type of setting is the continuous time model of Cox et al. (1985a), which has several applications in the literature (e.g. Cox et al. 1985b; Longstaff and Schwartz 1992, and Gagliardini et al. 2009 on the modeling of the term structure of interest rates). A pioneering example of the equilibrium approach is the model of Lucas (1978), which has a similar structure to that of Cox et al. (1985a), but has exogenous production, no technological change and is in discrete time. The model of Lucas (1978) has also several applications in the literature, with a recent example being the consumption based general equilibrium model for designing affine asset pricing models by Eraker and Shaliastovich (2008).

The arbitrage approach starts by assuming the dynamics of the state variables, of which the contingent claims depend, and an exogenous specification for the uncertainty prices. Then, by applying Itô's lemma and imposing the condition that there are no arbitrage opportunities, the prices of contingent claims are obtained. This is the standard approach in the option pricing literature (e.g. Black and Scholes 1973), with some exceptions (e.g. Amin and Ng 1993), and has been extensively used in other fields of finance. For example, Vasicek (1977) and Brennan and Schwartz (1979) used it to model the term structure of interest rates.

The equilibrium approach has clear advantages with respect to the arbitrage approach. As pointed out by Cox et al. (1985b), imposing exogenous uncertainty prices without any underlying economic equilibrium may lead to internal inconsistencies. In the equilibrium approach, uncertainty prices are endogenous and therefore part of the equilibrium. Moreover, models under the arbitrage approach say very little about the economic nature of the price of uncertainty.

In this paper, we consider a continuous time general equilibrium model for contingent claim pricing which is a two state variable version of the model of Cox et al. (1985a). It is assumed that the two state variables, $Y_{1 t}$ and $Y_{2 t}$, are correlated and both impact the expected return of the single production process, $Q_{t}$. Moreover, it is assumed that shocks in one of the state variables, $Y_{2 t}$, are correlated with those in the 
return of the production process, and that the representative agent is ambiguous about the stochastic process describing the dynamics of $Y_{2 t}$. Uncertainty in the model has therefore two dimensions: risk and ambiguity. ${ }^{1}$

Ambiguity about the stochastic process for the state variable $Y_{2 t}$ is introduced through a robust control approach. ${ }^{2}$ The representative agent considers contaminations, $P^{h}$, around a reference belief model, $P$. Aversion towards ambiguity is considered by assuming that, in the spirit of Gilboa and Schmeidler (1989), the agent chooses the worst possible contamination, i.e., the one associated with the lowest expected utility. ${ }^{3}$ It is found that ambiguity about $Y_{2 t}$ impacts the fundamental partial differential equation satisfied by the price of a contingent claim through two channels: the equilibrium uncertainty price associated with the ambiguous state variable, $Y_{2 t}$, and the equilibrium interest rate.

The specification for each of those channels, containing a risk and an ambiguity component, is obtained. Moreover, we conclude that the impact of ambiguity on the

\footnotetext{
${ }^{1}$ The distinction between risk and ambiguity was first pointed out by Knight (1921) and later supported by the empirical experiments of Ellsberg (1961) and others (see Camerer and Weber 1992 and Epstein and Schneider 2010 for a survey). The reason for this distinction is that economic agents may not be able to completely describe the uncertainty that they face by using a single probability distribution. Risk refers to uncertainty that can be represented by a probability distribution, while ambiguity refers to uncertainty that cannot. This distinction has relevant implications for the behavior of economic agents, and, therefore, for economic theory in general. That is why a rapidly growing literature on ambiguity aversion is emerging including, among others, macroeconomic topics such as business cycles and monetary policy (Hansen et al. 1999; Cagetti et al. 2002; Zhang and Semmler 2005; Ulrich 2010; Ilut and Schneider 2012), game theory topics (e.g. Eichberger and Kelsey 2011) and finance topics including optimal portfolio choice (e.g. Maenhout 2006; Garlappi et al. 2007; Ma et al. 2008; Liu 2010), non-participation or selective participation in markets (e.g. Cao et al. 2005; Ui 2011), equity premium and interest rate puzzles (e.g. Chen and Epstein 2002; Barillas et al. 2009), excess volatility puzzle (Leippold et al. 2008), home-bias puzzle (Epstein and Miao 2003; Uppal and Wang 2003) and American Options optimal exercise strategies (Riedel 2009). Asset pricing literature under ambiguity aversion has been comprehensively surveyed by Epstein and Schneider (2010)
}

2 An extensive review on decision theory under ambiguity has been carried out by Etner et al. (2012). Briefly, the two most common approaches being used in the ambiguity literature are: the robust control (RC) approach, associated to an assumption of model uncertainty (e.g. Maenhout 2004, 2006; Gagliardini et al. 2009); the multiple priors (MP) approach, from the seminal work by Gilboa and Schmeidler (1989), where the single probability measure of the standard expected utility model is replaced by a set of probabilities or priors. The relationship between the robust control and multiple priors approaches has been widely discussed in the literature (e.g. Hansen and Sargent 2001; Hansen et al. 2002; Epstein and Schneider 2003; Maccheroni et al. 2006).

${ }^{3}$ The approach of Gilboa and Schmeidler (1989) is sometimes criticized because it apparently implies extreme ambiguity aversion. However, the implied decision criteria may not be so extreme as it seems. The reasoning for this is that the set of priors is not an independent object including all logically possible priors, being instead part of the representation of the concrete problem under analysis. This is why the criteria of Gilboa and Schmeidler (1989) is not so extreme as, for example, the Wald maxmin criteria. As Epstein and Schneider (2010) claimed: "Ultimately, the only way to argue that the model is extreme is to demonstrate extreme behavioral implications of the axioms, something that has not been done". More recently, a smooth ambiguity aversion utility theory has been developed on the back of the seminal work of Klibanoff et al. (2005). It is claimed that this setup distinguishes ambiguity from ambiguity aversion and allows for smooth indifference curves, avoiding the infinite ambiguity aversion implied in the approach of Gilboa and Schmeidler (1989). However, there is still a debate in the literature about the axiomatic foundations of this line of models (see Epstein 2010 and Klibanoff et al. 2011 for a recent exchange on this). Also because of this, the approach of Gilboa and Schmeidler (1989) continues to be the main reference in the literature. 
equilibrium price of uncertainty associated with $Y_{2 t}$, and on the equilibrium interest rate depends on: (i) the correlations between the shocks in $Y_{2 t}$ and the shocks in the other state variable and in the output rate; (ii) the diffusion functions of the stochastic processes of $Y_{2 t}$ and of the economy's output rate; and (iii) the impact on utility of changes in $Y_{2 t}$.

This paper has two major contributions. The first is to develop a two-factor general equilibrium framework for asset pricing under ambiguity when the shocks in the two state variables are correlated but only the shocks in the ambiguous state variable are correlated with those of the economy's output rate. This is a simple setting that can be applied to many asset pricing problems. As an example, we apply our general results to the investment opportunity set of the well known option pricing model of Heston (1993), therefore providing an equilibrium motivation for the specification of the price of variance risk used there.

The second major contribution of the paper is the derivation of a variance uncertainty price specification used to obtain a closed form solution for option pricing with ambiguity about the stochastic variance process of the option's underlying asset return. This is carried out in Faria and Correia-da-Silva (2011). ${ }^{4}$

The paper is organized as follows. In Sect. 2, the intertemporal general equilibrium model for contingent claim pricing under ambiguity is developed. In Sect. 3, it is applied to a concrete investment opportunity set which contains that of the option pricing model of Heston (1993). In Sect. 4, we conclude the paper with some remarks.

\section{The model}

We consider an intertemporal general equilibrium model for contingent claim pricing that is a version of the model of Cox et al. (1985a) with two correlated state variables, a single stochastic constant returns-to-scale production process and logarithmic utility. ${ }^{5}$

There is a single physical good in the economy, that the representative agent can consume or reinvest in the stochastic production process, $Q_{t}$. The realized return on the physical investment made through the production process, i.e., the economy's output rate, is driven by two correlated state variables, $Y_{1 t}$ and $Y_{2 t}$ :

$$
\frac{d Q_{t}}{Q_{t}}=g_{Q}\left(Y_{1 t}, Y_{2 t}\right) d t+\sigma_{Q}\left(Y_{1 t}, Y_{2 t}\right) d W_{Q}
$$

where $g_{Q}\left(Y_{1 t}, Y_{2 t}\right)$ and $\sigma_{Q}\left(Y_{1 t}, Y_{2 t}\right)$ are generic expressions for the drift and diffusion functions of the output rate dynamics, which may depend on both state variables, and $W_{Q}$ is a standard Brownian motion.

\footnotetext{
4 We will build mostly on the work of Trojani and Vanini (2004) and Gagliardini et al. (2009). Other contributions to understanding the effects of ambiguity in general equilibrium settings are the seminal works of Dow and Werlang (1992) and Epstein and Wang (1994), and the more recent works of Correia-da-Silva and Hervés-Beloso (2009), de Castro et al. (2011) and Ozsoylev and Werner (2011).

5 We foresee as interesting extensions of this setting the consideration of heterogeneous agents, where trade would occur in equilibrium, and of a more general setting for preferences (e.g. SDU from Duffie and Epstein 1992a,b). We thank Frank Riedel for those suggestions for future work.
} 
The dynamics of the state variables, $\left(Y_{1 t}, Y_{2 t}\right)$, is given by:

$$
\begin{aligned}
& d Y_{1 t}=g_{Y_{1}}\left(Y_{1 t}, Y_{2 t}\right) d t+\sigma_{Y_{1}}\left(Y_{1 t}, Y_{2 t}\right) d W_{1}, \\
& d Y_{2 t}=g_{Y_{2}}\left(Y_{1 t}, Y_{2 t}\right) d t+\sigma_{Y_{2}}\left(Y_{1 t}, Y_{2 t}\right) d W_{2},
\end{aligned}
$$

where the generic functions $g_{Y_{1}}, \sigma_{Y_{1}}, g_{Y_{2}}$, and $\sigma_{Y_{2}}$ have the same meaning, for each of the state variables, as $g_{Q}$ and $\sigma_{Q}$ for the stochastic process (1). ${ }^{6}$ The processes $W_{1}$ and $W_{2}$ are standard Brownian motions with an instantaneous correlation equal to $\rho\left(d W_{1} d W_{2}=\rho d t\right)$.

Both state variables potentially impact the expected output rate, but it is assumed that only one of them, $Y_{2 t}$, has shocks that are correlated with those of the output rate. The instantaneous correlation between $\frac{d Q_{t}}{Q_{t}}$ and $d Y_{2 t}$ is $\rho_{2}\left(d W_{Q} d W_{2}=\rho_{2} d t\right)$.

The investment opportunity set given by (1), (2) and (3) can be described by the following system (Appendix 5.1):

$$
\left[\begin{array}{c}
\frac{d Q_{t}}{Q_{t}} \\
d Y_{1 t} \\
d Y_{2 t}
\end{array}\right]=\left[\begin{array}{c}
g_{Q} \\
g_{Y_{1}} \\
g_{Y_{2}}
\end{array}\right] d t+\underbrace{\left[\begin{array}{ccc}
\sigma_{Q} \sqrt{1-\frac{\rho_{2}^{2}}{1-\rho^{2}}} & 0 & \frac{\sigma_{Q} \rho_{2}}{\sqrt{1-\rho^{2}}} \\
0 & \sigma_{Y_{1}} & 0 \\
0 & \sigma_{Y_{2} \rho} & \sigma_{Y_{2}} \sqrt{1-\rho^{2}}
\end{array}\right]}_{\boldsymbol{A}}\left[\begin{array}{c}
d Z_{0} \\
d Z_{1} \\
d Z_{2}
\end{array}\right]
$$

where $Z_{i}(i=0,1,2)$ are independent Brownian motions. We assume that $\rho, \rho_{2} \in$ ] $-1,1$ [ (i.e., we exclude perfect correlations) and that $\rho_{2}<\sqrt{1-\rho^{2}}$ to guarantee that the elements of the matrix $\boldsymbol{A}$ are real numbers. For the presentation that follows, we make use of the following three matrices, $\boldsymbol{Z}, \boldsymbol{\sigma}$ and $\boldsymbol{\Xi}$ :

$$
\begin{aligned}
& \boldsymbol{Z}=\left[\begin{array}{c}
Z_{0} \\
Z_{1} \\
Z_{2}
\end{array}\right], \\
& \boldsymbol{\sigma}=\left[\begin{array}{lll}
\sigma_{Q} \sqrt{\left(1-\frac{\rho_{2}^{2}}{1-\rho^{2}}\right)} & 0 & \frac{\sigma_{Q} \rho_{2}}{\sqrt{1-\rho^{2}}}
\end{array}\right], \\
& \boldsymbol{\Xi}=\left[\begin{array}{lll}
0 & \sigma_{Y_{1}} & 0 \\
0 & \sigma_{Y_{2}} \rho & \sigma_{Y_{2}} \sqrt{1-\rho^{2}}
\end{array}\right] .
\end{aligned}
$$

\footnotetext{
${ }^{6}$ Diffusion functions $\sigma_{Q}, \sigma_{Y_{1}}$ and $\sigma_{Y_{2}}$ are assumed to be positive, as it is usual in the literature, due to the analogy with the statistical concept of standard deviation, although probabilistically it is not required so. We thank Paolo Porchia for this insight.
} 
Observe that $\boldsymbol{\Xi} \boldsymbol{\Xi}^{\top}$ represents the covariance matrix of the state variables $\left(Y_{1 t}, Y_{2 t}\right)$ :

$$
\boldsymbol{\Xi}^{\top}=\left[\begin{array}{cc}
\sigma_{Y_{1}}^{2} & \sigma_{Y_{2}} \sigma_{Y_{1}} \rho \\
\sigma_{Y_{2}} \sigma_{Y_{1}} \rho & \sigma_{Y_{2}}^{2}
\end{array}\right] .
$$

It is assumed that the representative agent is not totally sure about the data-generating processes (4) that characterize the investment opportunity set dynamics. This means that the uncertainty faced by the representative agent has two dimensions: risk and ambiguity.

Ambiguity about the investment opportunity set is introduced through a "constraint preferences" robust control approach, following the extension of the model of Cox et al. (1985b) made by Gagliardini et al. (2009).

It is assumed that the representative agent is ambiguous about the dynamics of $Y_{2 t}$. The agent considers contaminations (alternative models), $P^{h}$, around his reference belief, $P$. The contaminations are assumed to be absolutely continuous with respect to $P$, and, therefore, are equivalently described by contaminating drift processes, $\boldsymbol{h}$. In each of the alternative models, $P^{h}$, the Brownian motion becomes, therefore, $Z^{h}(t)=Z(t)+\int_{0}^{t} h(s) d s .^{7}$

Existence of ambiguity is analytically represented by perturbations of the drift, with respect to the reference belief, in the dynamics of the ambiguous state variable, $Y_{2 t}$. Aversion towards ambiguity is introduced by assuming that, in the spirit of Gilboa and Schmeidler (1989), the representative agent chooses from all the possible contaminations, $P^{h}$, the one that corresponds to the worst case scenario, i.e., the one associated with lower expected utility.

An upper bound is imposed on the contaminating drift processes, $\boldsymbol{h}$ :

$$
\boldsymbol{h}^{\top} \boldsymbol{h} \leqslant 2 \eta,
$$

where $\eta \geqslant 0$ is a parameter that can be interpreted as the level of ambiguity.

As highlighted by Gagliardini et al. (2009), the bound (6) should be such that alternative models are statistically close to the "reference belief" model: otherwise the agent would easily distinguish among them and, consequently, would not face ambiguity. That is, $\eta$ should be small. Moreover, the bound (6) constrains both the instantaneous time variation and the continuation value of the relative entropy between the reference belief, $P$, and any admissible contaminated belief, $P^{h}$. Trojani and Vanini (2004) explain that the set $\left\{\boldsymbol{h}: \boldsymbol{h}^{\top} \boldsymbol{h} \in[0,2 \eta], \forall t \geqslant 0\right\}$ defines a rectangular set of priors because any process $\boldsymbol{h}$ (and therefore any probability measure $P^{h}$ ) in this set corresponds to a selection of transition densities from $t$ to $t+d t, t \geqslant 0$, such that $\boldsymbol{h}^{\top} \boldsymbol{h} \in[0,2 \eta]$. The fact that the specification of the ambiguity aversion is based on

\footnotetext{
${ }^{7}$ Gagliardini et al. (2009) explain that, for tractability reasons, the analysis is restricted to the class of Markov-Girsanov kernels. The absolute continuity assumption between $P$ and $P^{h}$ guarantees the equivalence property between the probability measures and, consequently, that the Cameron-Martin-Girsanov theorem can be applied. Moreover, from this theorem and considering the diffusion family of models under consideration, all that a probability measure change implies is the change of the drift function of the stochastic processes.
} 
a rectangular set of priors guarantees a dynamically consistent preference ordering, and can be interpreted as a continuous time version of Epstein and Schneider (2003) Recursive Multiple Priors Utility. ${ }^{8}$ More generally, in Hansen and Sargent (2006) there is a comprehensive discussion of the dynamic consistency issue under the robust control approach.

Considering the system (4) that describes the investment opportunity set, ambiguity about $Y_{2 t}$ is introduced through contaminations of the Brownian motion $Z_{2}$. As in Gagliardini et al. (2009), for a two state-variable model, the admissible contaminating drift process is restricted to be $\boldsymbol{h}=\left[\begin{array}{lll}h_{0} & h_{1} & h_{2}\end{array}\right]^{\top}=\left[\begin{array}{lll}0 & 0 & h_{2}\end{array}\right]^{\top}$. The class of admissible Markovian drift contaminations satisfying this restriction and the entropy bound is denoted by $\mathcal{H}$.

Under an admissible contamination, $P^{h}$, the investment opportunity set is described by:

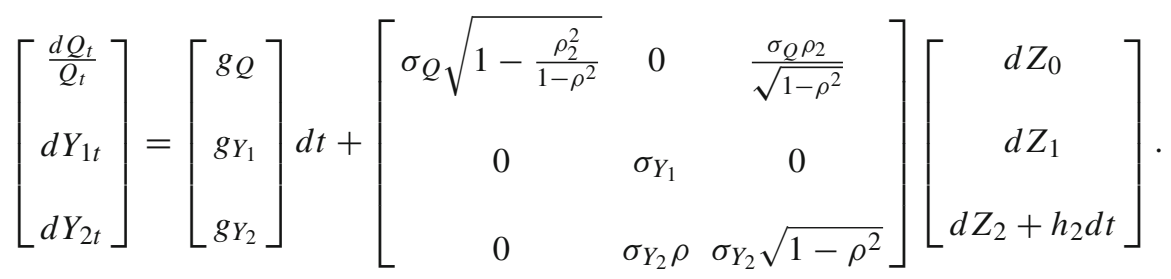

Note that in the "contaminated" system (7) that describes the investment opportunity set, the diffusion component continues to be driven by the same vector of independent Brownian motions, $\boldsymbol{Z}$ in (5). It is also straightforward to observe that the contamination $h_{2}$ only perturbs the drift functions in the stochastic processes of $\frac{d Q_{t}}{Q_{t}}$ and $d Y_{2 t}$, while keeping unchanged their diffusion functions.

The intertemporal budget constraint faced by the agent is given by:

$$
d W_{t}=W_{t} \frac{d Q_{t}}{Q_{t}}-C_{t} d t
$$

where $W_{t}$ and $C_{t}$ represent wealth and consumption at time $t$. Considering the output rate dynamics $\frac{d Q_{t}}{Q_{t}}$ in (7), the dynamic budget constraint can be expressed as:

$$
d W_{t}=\left(W_{t} g_{Q}-C_{t}\right) d t+W_{t} \sigma\left[\begin{array}{c}
d Z_{0} \\
d Z_{1} \\
d Z_{2}+h_{2} d t
\end{array}\right]
$$

with matrix $\sigma$ disclosed in (5).

\footnotetext{
8 See Epstein and Schneider (2003) for the definition of the rectangularity property. Additionally, in Trojani and Vanini (2004), p. 289, there is a detailed explanation supporting the rectangularity property of the present set of priors built under the constraint (6), and how this rectangular set of priors can be defined in the $k$-ignorance model of Chen and Epstein (2002).
} 
If the representative agent were not ambiguous about the dynamics of $Y_{2 t}$, then his problem would be to find the optimal consumption strategy, $C:\left[0,+\infty\left[\rightarrow \mathbb{R}_{+}\right.\right.$, that maximizes his expected intertemporal utility. As in the setting of Cox et al. (1985a), the optimal consumption strategy is financed by allocating all the wealth in the production process and none in the financial assets (which are in zero net supply). This implies that the only relevant control variable, for the non-ambiguous agent, is the consumption flow process.

However, with the representative agent being ambiguous about the dynamics of $Y_{2 t}$, there isn't a single probability measure, $P$, to be considered when assessing his expected utility. Instead, a set of probability measures, $P^{h}$, has to be considered. The existence of ambiguity therefore implies that the solution of the representative agent's problem also involves solving for the most adverse contaminating drift process $\boldsymbol{h} \in \mathcal{H}$.

Having a logarithmic instantaneous utility function, the ambiguity averse representative agent solves the following Maxmin expected utility program:

$$
J\left(W_{0}, Y_{10}, Y_{20}\right)=\sup _{C} \inf _{h \in \mathcal{H}} E^{h}\left[\int_{0}^{\infty} e^{-\delta s} \ln \left(C_{s}\right) d s\right],
$$

subject to the dynamics of state variables $Y_{1 t}$ and $Y_{2 t}$, represented in (7), and to the dynamic budget constraint (8). The operator $E^{h}$ denotes expectations under the measure $P^{h}, \delta>0$ is the subjective rate of discount of the representative agent, and $J\left(W_{0}, Y_{10}, Y_{20}\right)=\left.J\left(W_{t}, Y_{1 t}, Y_{2 t}\right)\right|_{t=0}$ denotes the value function of the problem.

Applying Proposition 1 in Gagliardini et al. (2009), the value function of the ambiguity-averse agent is given by:

$$
J\left(W_{0}, Y_{10}, Y_{20}\right)=-\frac{1}{\delta}+\frac{\ln \left(\delta W_{0}\right)}{\delta}+\frac{1}{\delta} V\left(Y_{10}, Y_{20}\right),
$$

where

$$
V\left(Y_{10}, Y_{20}\right)=\inf _{h \in \mathcal{H}} E^{h}\left[\int_{0}^{\infty} e^{-\delta s}\left(g_{Q}-\frac{1}{2} \sigma \sigma^{\top}+\sigma h_{s}\right) d s\right],
$$

subject to $d Y_{1 t}$ and $d Y_{2 t}$ in (7) and with $\sigma$ given by (5). The corresponding Bellman equation solved by the value function $V\left(Y_{10}, Y_{20}\right)$ is given by:

$$
\begin{aligned}
0= & \boldsymbol{V}_{\boldsymbol{Y}}^{\top} \boldsymbol{g}_{\boldsymbol{Y}}+\frac{1}{2} \operatorname{trace}\left[\boldsymbol{\Xi}^{\top} \boldsymbol{V}_{\boldsymbol{Y} \boldsymbol{Y}} \boldsymbol{\Xi}\right]-\sqrt{2 \eta} \sqrt{\left(\boldsymbol{\Xi}^{\top} \boldsymbol{V}_{\boldsymbol{Y}}+\boldsymbol{\sigma}^{\top}\right)^{\top}\left(\boldsymbol{\Xi}^{\top} \boldsymbol{V}_{\boldsymbol{Y}}+\boldsymbol{\sigma}^{\top}\right)} \\
& +g_{Q}-\frac{1}{2} \boldsymbol{\sigma} \boldsymbol{\sigma}^{\top}-\delta V,
\end{aligned}
$$

where $V_{Y}$ and $V_{Y Y}$ are the gradient and Hessian matrices of the value function $V\left(Y_{10}, Y_{20}\right)$ with respect to the state variables; ${ }^{9}$ and $\boldsymbol{g}_{\boldsymbol{Y}}$ is the vector of drift functions of the state variables.

\footnotetext{
9 In general, it is known that a value function may not be differentiable, at least in the entire domain of the state variables. If the differentiability property is not satisfied then the "viscosity solution" of the stochastic optimal control problem has to be studied.
} 
The equilibrium contamination drift vector, $\boldsymbol{h}^{*}=\left[\begin{array}{lll}0 & 0 & h_{2}\end{array}\right]^{\top}$, that solves the model selection problem is obtained directly from Proposition 1 in Gagliardini et al. (2009), with equilibrium $h_{2}$ being given by (Appendix 5.2):

$$
h_{2}= \begin{cases}-\sqrt{2 \eta} & \text { if } V_{Y_{2}}>-\frac{\sigma_{Q} \rho_{2}}{\sigma_{Y_{2}}\left(1-\rho^{2}\right)}, \\ \sqrt{2 \eta} & \text { if } V_{Y_{2}}<-\frac{\sigma_{Q} \rho_{2}}{\sigma_{Y_{2}}\left(1-\rho^{2}\right)},\end{cases}
$$

where $V_{Y_{2}}$ represents the gradient of the value function $V\left(Y_{1 t}, Y_{2 t}\right)$ in (11), with respect to the ambiguous state variable $Y_{2 t}$. The validity of this expression is guaranteed by previous assumptions of $\rho \in]-1,1\left[\right.$ and $\sigma_{Y_{2}}>0$ (non-deterministic state variable).

From the Cameron-Martin-Girsanov theorem, coupled with the fact that in our setting only diffusion models are considered, it results that the change from one probability measure to an equivalent probability measure only leads to a change of drift in the stochastic processes of the state variables. Considering the reference belief $P$ and an equivalent "uncertainty-neutralized" probability measure, then the change of drift associated with each of the state variables, represented by the matrix $\phi=\left[\begin{array}{ll}\phi_{1} & \phi_{2}\end{array}\right]^{\top}$, is the equilibrium price of uncertainty associated to each of the state variables.

In the present setting, the equilibrium prices of uncertainty associated to $Y_{1 t}$ and $Y_{2 t}$ are given by (Appendix 5.2):

$$
\begin{aligned}
& \phi_{1}=0 \\
& \phi_{2}= \begin{cases}\sigma_{Y_{2}} \sigma_{Q} \rho_{2}+\sigma_{Y_{2}} \sqrt{2 \eta} \sqrt{1-\rho^{2}} & \text { if } V_{Y_{2}}>-\frac{\sigma_{Q} \rho_{2}}{\sigma_{Y_{2}}\left(1-\rho^{2}\right)}, \\
\sigma_{Y_{2}} \sigma_{Q} \rho_{2}-\sigma_{Y_{2}} \sqrt{2 \eta} \sqrt{1-\rho^{2}} & \text { if } V_{Y_{2}}<-\frac{\sigma_{Q} \rho_{2}}{\sigma_{Y_{2}}\left(1-\rho^{2}\right)},\end{cases}
\end{aligned}
$$

respectively.

The result in (14) means that, in equilibrium, uncertainty about $Y_{1 t}$ has a null price. This should not be a surprise, considering that there is no ambiguity about the dynamics of $Y_{1}$ and that its shocks are uncorrelated with those of the output rate. Regarding the state variable $Y_{2 t}$, there exists an associated equilibrium price of uncertainty, given by (15), as its shocks are correlated with those of economy's output rate (implying an equilibrium price of risk) and the representative agent is ambiguous about its stochastic process (implying an equilibrium price of ambiguity).

In fact, from (15), it is clear that the equilibrium price of uncertainty associated with $Y_{2 t}$ is divided in two components: the equilibrium price of risk, given by $\sigma_{Y_{2}} \sigma_{Q} \rho_{2}$, and the equilibrium price of ambiguity, given by $\pm \sigma_{Y_{2}} \sqrt{2 \eta} \sqrt{\left(1-\rho^{2}\right)}$.

We therefore conclude that the existence of ambiguity about $Y_{2 t}$ implies an additional equilibrium price component. From (15), it results that such component can be positive or negative. Consequently, the equilibrium uncertainty price associated with that state variable, $Y_{2 t}$, can either increase or decrease when the agent is ambiguous 
about its stochastic process, depending on the relation between: (i) the impact on the indirect utility (value function) of changes in the ambiguous state variable $\left(V_{Y_{2}}\right)$; (ii) the correlations between shocks in that variable and in the other state variable $(\rho)$ as well as in the economy's output rate $\left(\rho_{2}\right)$; and (iii) the diffusion functions of the stochastic processes for the ambiguous state variable $\left(\sigma_{Y_{2}}\right)$ and the economy's output rate $\left(\sigma_{Q}\right)$.

Note also that the uncertainty price becomes preference-dependent when ambiguity aversion is considered (as it includes the $\eta$ parameter). This does not happen when uncertainty is exclusively risk.

In order to derive the partial differential equation (PDE) satisfied by the contingent claim price under the present setting, it is still necessary to obtain the equilibrium instantaneous interest rate, $r_{t}$. The generic expression for $r_{t}$ is given by (Appendix 5.2):

$$
r_{t}= \begin{cases}g_{Q}-\sigma_{Q}^{2}-\frac{\sigma_{Q} \rho_{2}}{\sqrt{1-\rho^{2}}} \sqrt{2 \eta} & \text { if } V_{Y_{2}}>-\frac{\sigma_{Q} \rho_{2}}{\sigma_{Y_{2}}\left(1-\rho^{2}\right)} \\ g_{Q}-\sigma_{Q}^{2}+\frac{\sigma_{Q} \rho_{2}}{\sqrt{1-\rho^{2}}} \sqrt{2 \eta} & \text { if } V_{Y_{2}}<-\frac{\sigma_{Q} \rho_{2}}{\sigma_{Y_{2}}\left(1-\rho^{2}\right)}\end{cases}
$$

where the first two parcels $\left(g_{Q}-\sigma_{Q}^{2}\right)$, give the equilibrium instantaneous interest rate when uncertainty is exclusively risk $(\eta=0)$, and the third parcel $\left( \pm \frac{\sigma_{Q} \rho_{2}}{\sqrt{1-\rho^{2}}} \sqrt{2 \eta}\right)$ is the new component that results from the existence of ambiguity. The expression for the equilibrium interest rate when uncertainty is exclusively risk, given by the difference between the expected output rate $\left(g_{Q}\right)$ and the variance of the output rate $\left(\sigma_{Q}^{2}\right)$, is consistent with findings in the literature based on the setting of Cox et al. (1985a), without ambiguity (e.g. in Longstaff and Schwartz 1992). ${ }^{10}$

Moreover, looking at the ambiguity component in (16), we conclude that ambiguity about the stochastic process of $Y_{2 t}$ does not impact the equilibrium interest rate when the economy's output rate is deterministic $\left(\sigma_{Q}=0\right)$ or when shocks in the ambiguous state variable are uncorrelated with those of the output rate $\left(\rho_{2}=0\right)$. In general, the equilibrium instantaneous interest rate under ambiguity, $r_{t}$, can be higher or lower than when uncertainty is exclusively risk, depending on the sign of $\rho_{2}$ and, as in (15), on the relation between: (i) the impact on the indirect utility (value function) of changes in the ambiguous state variable $\left(V_{Y_{2}}\right)$; (ii) the correlations between shocks in that variable and in the other state variable $(\rho)$ as well as in the economy's output rate $\left(\rho_{2}\right)$; and

\footnotetext{
10 Regarding (16), depending on the specifications of $g_{Q}$ and $\sigma_{Q}$, conditions on parameters have to be imposed in order to guarantee that the equilibrium interest rate is non-negative. As explained by Longstaff and Schwartz (1992), the lower bound of zero for the interest rate is consistent with the basic properties of the economy under study, because as the single good produced in this economy can be consumed or invested in the production process, it can be seen as storable.
} 
(iii) the diffusion functions of the stochastic processes for the ambiguous state variable $\left(\sigma_{Y_{2}}\right)$ and economy's output rate $\left(\sigma_{Q}\right){ }^{11}$

Given the equilibrium price of uncertainty associated with $Y_{1 t}$ and $Y_{2 t}$, (14) and (15) respectively, and the equilibrium instantaneous interest rate (16), from Proposition 2 in Gagliardini et al. (2009) the fundamental partial differential equation (PDE) satisfied by the price of a contingent claim with maturity $\tau, H\left(Y_{1 t}, Y_{2 t}, \tau\right)$, assumed to depend on both state variables but not on wealth, is given by:

$$
\begin{aligned}
\frac{\partial H}{\partial \tau}= & \frac{1}{2} \sigma_{Y_{1}}^{2}\left(Y_{1 t}, Y_{2 t}\right) \frac{\partial^{2} H}{\partial Y_{1}^{2}}+\frac{1}{2} \sigma_{Y_{2}}^{2}\left(Y_{1 t}, Y_{2 t}\right) \frac{\partial^{2} H}{\partial Y_{2}^{2}} \\
& +\rho \sigma_{Y_{1}}\left(Y_{1 t}, Y_{2 t}\right) \sigma_{Y_{2}}\left(Y_{1 t}, Y_{2 t}\right) \frac{\partial^{2} H}{\partial Y_{1} \partial Y_{2}}+\left[g_{Y_{1}}\left(Y_{1 t}, Y_{2 t}\right)-\phi_{1}\right] \frac{\partial H}{\partial Y_{1}} \\
& +\left[g_{Y_{2}}\left(Y_{1 t}, Y_{2 t}\right)-\phi_{2}\right] \frac{\partial H}{\partial Y_{2}}-r_{t} H+\Lambda\left(W_{t}, Y_{1 t}, Y_{2 t}, t\right),
\end{aligned}
$$

where (i) $\phi_{1}, \phi_{2}$ and $r_{t}$ are given by (14), (15) and (16), respectively and (ii) $\Lambda\left(W_{t}, Y_{1 t}, Y_{2 t}, t\right)$ represents the instantaneous payoff of the contingent claim, which depends on its specific contractual conditions.

From the PDE (17), it is clear that ambiguity aversion impacts the fundamental pricing equation through two preference-dependent inputs: the equilibrium instantaneous interest rate and the equilibrium price of uncertainty associated with the ambiguous state variable $Y_{2 t}$.

A particular case of this setting is presented in Longstaff and Schwartz (1992), where an intertemporal general equilibrium setting for valuing interest rate sensitive contingent claims is developed starting from a two state variable version of the model of Cox et al. (1985a). There, it is also assumed that both state variables impact the expected output rate in the economy, that only one of them has shocks correlated with those of the economy's output rate, and that the representative agent has a logarithmic utility function. They assume, however, that the state variables are uncorrelated. Moreover, in their setting, uncertainty is exclusively risk, i.e., there is no ambiguity. The model of Longstaff and Schwartz (1992) is, therefore, a particular case of our setting (see Appendix 5.3).

\section{An example}

In the previous section, we developed, for a general investment opportunity set with endogenous production driven by two correlated state variables, an intertemporal equilibrium setting for contingent claim pricing following Cox et al. (1985a) and Gagliardini et al. (2009), considering that uncertainty includes two dimensions: risk

\footnotetext{
11 As highlighted in Epstein and Schneider (2010), agent's willingness to save is a positive function of his level of uncertainty and the more the agent tries to save the lower tends to be the equilibrium interest rate. Considering ambiguity as an extra source of uncertainty (alongside risk), we may therefore conclude that the most intuitive scenario is that when ambiguity is considered, the equilibrium interest rate decreases (everything else constant). This is also the result, e.g., under the general equilibrium model with ambiguity in Trojani and Vanini (2004) and Xu et al. (2011).
} 
and ambiguity. A key characteristic of our investment opportunity set is that the state variables, $Y_{1 t}$ and $Y_{2 t}$, are correlated but only one of them, $Y_{2 t}$, has shocks correlated with those of the economy's output rate, and, simultaneously, the representative agent is ambiguous about its stochastic process.

In this section, as an example, we apply results of the previous section by considering a concrete investment opportunity set, where the state variable $Y_{1 t}$ is an economic variable and $Y_{2 t}$ represents the variance of changes in $Y_{1 t}$.

The return of the economy's production process (output rate) is assumed to be given by:

$$
\frac{d Q_{t}}{Q_{t}}=g_{Q}\left(Y_{1 t}, Y_{2 t}\right) d t+l \sqrt{Y_{2 t}} d W_{Q},
$$

where $g_{Q}$ is the drift function and $l>0$.

It is assumed that $Y_{1 t}$ follows the geometric Brownian motion:

$$
d Y_{1 t}=\mu Y_{1 t} d t+Y_{1 t} \sqrt{Y_{2 t}} d W_{1},
$$

where $\mu$ is the expected growth rate of $Y_{1 t}, Y_{2 t}$ is its instantaneous variance and it is assumed that $d W_{Q} d W_{1}=0$.

Regarding the ambiguous state variable, $Y_{2 t}$, the "reference belief" dynamics for the representative agent is assumed to be given by the mean-reverting square-root process (as used, for example, in Cox et al. 1985b):

$$
d Y_{2 t}=\kappa\left(\theta-Y_{2 t}\right) d t+\epsilon \sqrt{Y_{2 t}} d W_{2}
$$

where $\theta$ is the expected value of $Y_{2 t}, \kappa>0$ is the mean-reverting parameter and $\epsilon>0$. It is assumed that $d W_{1} d W_{2}=\rho d t$ and $d W_{Q} d W_{2}=\rho_{2} d t$.

From (18), (19) and (20), we have $\sigma_{Q}=l \sqrt{Y_{2 t}}, \sigma_{Y_{1}}=Y_{1 t} \sqrt{Y_{2 t}}$ and $\sigma_{Y_{2}}=\epsilon \sqrt{Y_{2 t}}$, therefore, from (15), it is straightforward to obtain the specification of $\phi_{2}$, the equilibrium market price of uncertainty associated with $Y_{2 t}$ :

$$
\phi_{2}=\underbrace{\epsilon l \rho_{2} Y_{2 t} t}_{\text {risk price }} \underbrace{ \pm \epsilon \sqrt{2 \eta\left(1-\rho^{2}\right) Y_{2 t}}}_{\text {ambiguity price }},
$$

which, by defining $\lambda_{1}=\epsilon l \rho_{2}$ and $\lambda_{2}= \pm \epsilon \sqrt{2 \eta\left(1-\rho^{2}\right)}$, can be written as:

$$
\phi_{2}=\lambda_{1} Y_{2 t}+\lambda_{2} \sqrt{Y_{2 t}} \text {. }
$$

The equilibrium market price of uncertainty associated with the variance of changes in $Y_{1 t}$ has two components: the variance risk price, which is linear on the instantaneous level of variance, $Y_{2 t}$, and the variance ambiguity price, which is proportional to the square-root of $Y_{2 t}$. The variance risk price depends on: (i) the parameter $l$ of the diffusion function in the stochastic process describing the economy's output rate; 
(ii) the parameter $\epsilon$ of the diffusion function of the stochastic process of $Y_{2 t}$; and (iii) on the correlation $\rho_{2}$ between shocks in $d Y_{2 t}$ and in the output rate. The variance risk price is positive (negative) when $\rho_{2}>0\left(\rho_{2}<0\right)$, since, by assumption, $\epsilon>0, l>0$ (see also footnote 6 ). The variance ambiguity price depends on $\epsilon$, on the correlation of shocks in both state variables $(\rho)$, and on the degree of ambiguity faced by the representative agent $(\eta)$. It can also be positive or negative.

Note that the specification for the dynamics of the state variables (19) and (20) is the one that is used in Heston (1993) stochastic volatility option pricing model. In Heston (1993), $Y_{1 t}$ represents the option's underlying asset spot price and, consequently, $Y_{2 t}$ is the variance of the underlying asset return, with both being correlated. The specification for the market price of variance risk used in Heston (1993), where uncertainty is exclusively risk, is a scalar multiplied by the instantaneous level of variance. This is consistent with our findings under the developed equilibrium approach: it corresponds to the $\lambda_{1} Y_{2 t}$ component in (21). We have therefore provided an equilibrium motivation for the price specification of variance risk in Heston (1993) model. We also conclude that a potential extension of Heston (1993) model by incorporating ambiguity aversion about the stochastic variance process of the underlying asset return could use (21) as the specification for the variance uncertainty price, with the sign of the ambiguity component depending on the concrete calibration to be used.

From (16), it is straightforward to obtain the expression for the equilibrium interest rate:

$$
r_{t}=g_{Q}-l^{2} Y_{2 t} \pm \frac{l \rho_{2}}{\sqrt{1-\rho^{2}}} \sqrt{2 \eta} \sqrt{Y_{2 t}}
$$

where the new component emerging from the ambiguity consideration is $\pm \frac{l \rho_{2}}{\sqrt{1-\rho^{2}}}$ $\sqrt{2 \eta} \sqrt{Y_{2 t}}$.

In order to study the sign of the ambiguity components in both (21) and (22), we must specify the output rate drift function $g_{Q}$ and, subsequently, solve the corresponding Bellman equation (12). This is illustrated in the next subsection.

\subsection{A particular solution}

Assuming $g_{Q}\left(Y_{1 t}, Y_{2 t}\right)=\ln Y_{1 t}+\alpha Y_{2 t}$, where $\alpha$ is a scalar parameter, the output rate process (18) is given by:

$$
\frac{d Q_{t}}{Q_{t}}=\left(\ln Y_{1 t}+\alpha Y_{2 t}\right) d t+l \sqrt{Y_{2 t}} d W_{Q}
$$

Considering this concrete specification for the output rate process and the processes (19) and (20) for the state variables, we start by solving the corresponding Bellman equation (12), which is given by (Appendix 5.4.1): 


$$
\begin{aligned}
\delta V= & \mu V_{Y_{1}} Y_{1 t}+\kappa\left(\theta-Y_{2 t}\right) V_{Y_{2}}+\ln Y_{1 t}+\alpha Y_{2 t}-\frac{1}{2} l^{2} Y_{2 t} \\
& +\frac{1}{2}\left(Y_{1 t}^{2} Y_{2 t} V_{Y_{1} Y_{1}}+2 \epsilon \rho V_{Y_{2} Y_{1}} Y_{1 t} Y_{2 t}+\epsilon^{2} Y_{2 t} V_{Y_{2} Y_{2}}\right)-\sqrt{2 \eta F\left(Y_{1 t}, Y_{2 t}\right)}
\end{aligned}
$$

where

$$
\begin{aligned}
F\left(Y_{1 t}, Y_{2 t}\right)= & l^{2} Y_{2 t}\left(1-\frac{\rho_{2}^{2}}{1-\rho^{2}}\right)+\left(V_{Y_{1}} Y_{1 t} \sqrt{Y_{2 t}}+\epsilon \sqrt{Y_{2 t}} \rho V_{Y_{2}}\right)^{2} \\
& +\left(\epsilon \sqrt{Y_{2 t}} \sqrt{1-\rho^{2}} V_{Y_{2}}+\frac{l \sqrt{Y_{2 t}} \rho_{2}}{\sqrt{1-\rho^{2}}}\right)^{2} .
\end{aligned}
$$

We obtain a solution that is exact when there is no ambiguity $(\eta=0)$ and approximate in the presence of ambiguity (Appendix 5.4.2):

$$
V\left(Y_{1 t}, Y_{2 t}\right)=a \ln Y_{1 t}+b Y_{2 t}+c,
$$

with

$$
a=\frac{1}{\delta}, \quad b=\frac{\left(\alpha-\frac{l^{2}}{2}-\frac{1}{2 \delta}\right)}{(\kappa+\delta)} \text { and } c=\frac{\mu}{\delta^{2}}+\frac{\kappa \theta}{\delta} b .
$$

The value function (25) is an approximate solution of (24) in the domain $0<\eta<\Psi$ (with $\Psi$ being an arbitrarily small positive number), assuming that $V_{Y_{2}}$ and $\frac{\partial V_{Y_{2}}}{\partial \eta}$ exist. It is difficult to obtain an exact solution for (24) under ambiguity $(\eta>0)$. We suspect that, if a solution exists, it is not separable in the state variables, making it difficult to study its gradient with respect to $Y_{2 t}$, and numerical procedures are necessary to find it. Moreover, there is a reason to believe that the accuracy of the approximation is reasonable: the domain $0<\eta<\Psi$ must be very tight, for the reasoning previously invoked that alternative models must be statistically close to the "reference belief" model, so that the representative agent has difficulty to distinguish them and therefore faces ambiguity.

This asymptotic method of finding an approximate solution of the problem is intuitively close to the perturbation theory used in Trojani and Vanini (2004) to solve intertemporal general equilibrium models under ambiguity. The rationale is provided by the authors (p. 291) "the basic idea of asymptotic methods is to formulate a general problem, find a particular relevant case that has a known solution, and use this as a starting point for computing the solution to nearby problems". As in our case, in Trojani and Vanini (2004)), the asymptotic solutions of the problems under ambiguity "...hold for neighborhoods of a model with log utility of consumption and no ambiguity aversion”. 
Table 1 Impact on $\phi_{2}$ and $r_{t}$ from ambiguity about the stochastic process of $Y_{2 t}$

\begin{tabular}{lllll}
\hline & $\alpha>\omega$ & & $\alpha<\omega$ & \\
\cline { 2 - 5 } & $\rho_{2}<0$ & $\rho_{2}>0$ & $\rho_{2}<0$ & $\rho_{2}>0$ \\
\hline$\phi_{2}$ & $\uparrow$ & $\uparrow$ & $\downarrow$ & $\downarrow$ \\
$r_{t}$ & $\uparrow$ & $\downarrow$ & $\downarrow$ & $\uparrow$ \\
\hline
\end{tabular}

The sign $\uparrow(\downarrow)$ indicates that ambiguity about $Y_{2 t}$ stochastic process increases (decreases) $\phi_{2}$ or $r_{t}$

From (25), it is immediate that $V_{Y_{2}}=b$, and the expression for the equilibrium price of uncertainty associated with $Y_{2}$ (21) can be clarified (Appendix 5.4.3):

$$
\phi_{2}=\lambda_{1} Y_{2 t}+\lambda_{2} \sqrt{Y_{2 t}}, \text { with } \lambda_{2}>0(<0) \text { if } \alpha>\omega(<\omega)
$$

where the threshold value $\omega$ is given by:

$$
\omega=\frac{\left(l^{2} \delta \epsilon+\epsilon\right)\left(1-\rho^{2}\right)-2 \delta(\kappa+\delta) l \rho_{2}}{2 \delta \epsilon\left(1-\rho^{2}\right)} .
$$

The equilibrium uncertainty price associated with $Y_{2 t}$ can therefore increase or decrease when ambiguity about its process is considered. That depends on the relative magnitude of the parameter $\alpha$, which measures the sensitivity of the expected output rate of the economy relatively to changes on $Y_{2 t}$, versus a benchmark value that synthesizes some information of the investment opportunity set (parameters $l, \epsilon, \kappa, \rho$ and $\rho_{2}$ ) and the subjective rate of discount of the representative agent, $\delta$. If $\alpha>\omega$, ambiguity about the stochastic process of $Y_{2 t}$ increases its equilibrium uncertainty price (and the contrary when $\alpha<\omega$ ).

Regarding the expression for the equilibrium instantaneous interest rate, $r_{t}$, in this concrete setting, from (16), it is given by (Appendix 5.4.3):

$$
r_{t}= \begin{cases}\left(\ln Y_{1 t}+\alpha Y_{2 t}\right)-l^{2} Y_{2 t}-\frac{l \rho_{2}}{\sqrt{1-\rho^{2}}} \sqrt{2 \eta} \sqrt{Y_{2 t}} & \text { if } \alpha>\omega, \\ \left(\ln Y_{1 t}+\alpha Y_{2 t}\right)-l^{2} Y_{2 t}+\frac{l \rho_{2}}{\sqrt{1-\rho^{2}}} \sqrt{2 \eta} \sqrt{Y_{2 t}} & \text { if } \alpha<\omega .\end{cases}
$$

From (28), one concludes that if there exists a negative correlation between shocks in the ambiguous state variable and the economy's output rate $\left(\rho_{2}<0\right)$, the impact on the equilibrium interest rate from ambiguity has the same direction (increase or decrease) as on the equilibrium uncertainty price (the contrary happens when $\rho_{2}>0$ ).

Overall, the effects on $\phi_{2}$ and $r_{t}$ from the consideration of ambiguity about the stochastic process of $Y_{2 t}$ are summarized in Table 1. 


\section{Concluding remarks}

We developed a general intertemporal equilibrium setting for asset pricing using a two state variable version of the model of Cox et al. (1985a). All the physical investment is delivered by a single stochastic production process whose realized return (economy's output rate) is driven by two state variables, $Y_{1 t}$ and $Y_{2 t}$. It is assumed that both state variables impact the economy's expected output rate, but only one of them $\left(Y_{2 t}\right)$ has shocks correlated with those of the output rate. A key assumption in our setting is that the state variables are correlated, which we believe to be quite useful for modeling economic problems, particularly regarding asset pricing.

It is assumed that the representative agent, with a logarithmic utility function, is not totally sure about the probability measure $P$ under which his investment opportunity set evolves. More, precisely, it is assumed that the representative agent is ambiguous about the stochastic model that characterizes the dynamics of the state variable $Y_{2 t}$. The representative agent considers contaminations around his reference belief and aversion towards ambiguity is introduced by assuming that, in the spirit of Gilboa and Schmeidler (1989), the representative agent chooses from all the contaminations the one associated with lower expected utility. Ambiguity aversion changes the fundamental pricing equation satisfied by the contingent claim price through two inputs, that become preference-dependent: the equilibrium instantaneous interest rate and the equilibrium price of uncertainty associated with $Y_{2 t}$. Those two inputs embed two components, corresponding to the two uncertainty dimensions: risk and ambiguity.

It is found that the equilibrium market price of risk associated with the state variable $Y_{2 t}$ depends on its correlation with the economy's output rate shocks and on the diffusion functions of the stochastic processes of the output rate and of $Y_{2 t}$. The equilibrium interest rate, when uncertainty is exclusively risk, is found to be given by the difference between the expected output rate of the economy and the variance of the output rate. Longstaff and Schwartz (1992) general equilibrium model for the interest rate term structure can be obtained as a particular case of our setting.

When ambiguity is considered, the equilibrium price of uncertainty associated with $Y_{2 t}$ and the equilibrium interest rate can increase or decrease, depending on (i) the impact of changes in the ambiguous state variable, $Y_{2 t}$, on the indirect utility (value function); (ii) the correlations between shocks in $Y_{2 t}$ and shocks in the other state variable, $Y_{1 t}$, and in the economy's output rate; and (iii) the diffusion functions of the stochastic processes for the ambiguous state variable, $Y_{2 t}$, and the economy's output rate.

As an example, we apply the obtained general results to a specific investment opportunity set, where $Y_{2 t}$ is the instantaneous variance of the change of $Y_{1 t}$, continuing to assume that both are correlated but only $Y_{2 t}$ has shocks correlated with those of economy's output rate. This contains the investment opportunity set of the well known option pricing model of Heston (1993), by letting $Y_{1 t}$ be the option's underlying asset price. The obtained equilibrium market price of variance risk is linear on its instantaneous level, which in fact is the specification used by Heston (1993): we therefore provide an equilibrium motivation for the specification of the price of variance risk used by Heston (1993). The obtained equilibrium market price of ambiguity about stochastic variance is proportional to the square-root of its instantaneous level. 
Through this example, we have therefore obtained a specification of the equilibrium variance uncertainty price that can be used in an extension of Heston (1993) model that accommodates ambiguity aversion about the stochastic variance process of the option's underlying asset return. This extension is carried out in Faria and Correia-daSilva (2011).

Acknowledgments We are grateful to Alejandro Balbás, Cláudia Ribeiro, Frank Riedel, Paolo Porchia, Tony Berrada, and an anonymous referee for useful comments and suggestions, and thank seminar participants in the 6th Portuguese Finance Network Conference, in the 4th Economic Theory Workshop in Vigo and in the 20th European Workshop on General Equilibrium Theory. Gonçalo Faria acknowledges support from FCT_-Fundação para a Ciência e Tecnologia (SFRH/BPD/74020/2010) and RGEA (Research Group in Economic Analysis-Universidad de Vigo). João Correia-da-Silva acknowledges support from CEF.UP, FCT and FEDER (research grant PTDC/EGE-ECO/111811/2009).

\section{Appendix}

\subsection{Correlation structure}

In the setting of Cox et al. (1985a) the Brownian motions that impact the dynamics of the output rate and the state variables are assumed to be independent. It is possible to rewrite the diffusion component of the system (1), (2) and (3) in a way that, maintaining the desired correlation structure, there is a vector of independent Brownian motions and, consequently, making it possible to apply the results of Cox et al. (1985a).

The diffusion component of the system (1), (2) and (3) is given by:

$$
\left[\begin{array}{ccc}
\sigma_{Q} & 0 & 0 \\
0 & \sigma_{Y_{1}} & 0 \\
0 & 0 & \sigma_{Y_{2}}
\end{array}\right]\left[\begin{array}{l}
d W_{Q} \\
d W_{1} \\
d W_{2}
\end{array}\right]
$$

where (i) $d W_{Q} d W_{1}=0, d W_{Q} d W_{2}=\rho_{2} d t$ and $d W_{1} d W_{2}=\rho d t$, (ii) the variance of the output rate, of $d Y_{1 t}$ and of $d Y_{2 t}$ is given by $\sigma_{Q}^{2}, \sigma_{Y_{1}}^{2}$ and $\sigma_{Y_{2}}^{2}$, respectively and, consequently, (iii) $\operatorname{Cov}\left(\frac{d Q_{t}}{Q_{t}}, Y_{1 t}\right)=0, \operatorname{Cov}\left(\frac{d Q_{t}}{Q_{t}}, Y_{2 t}\right)=\sigma_{Q} \sigma_{Y_{2 t}} \rho_{2}$ and $\operatorname{Cov}\left(Y_{1 t}, Y_{2 t}\right)=\sigma_{Y_{1 t}} \sigma_{Y_{2 t}} \rho$, where $\operatorname{Cov}(\cdot)$ stands for the covariance.

In order to maintain this correlation structure when considering the vector $\boldsymbol{Z}$ of independent Brownian motions in (5), it is necessary that:

$$
\left[\begin{array}{ccc}
\sigma_{Q} & 0 & 0 \\
0 & \sigma_{Y_{1}} & 0 \\
0 & 0 & \sigma_{Y_{2}}
\end{array}\right]\left[\begin{array}{l}
d W_{Q} \\
d W_{1} \\
d W_{2}
\end{array}\right]=\boldsymbol{A}\left[\begin{array}{l}
d Z_{0} \\
d Z_{1} \\
d Z_{2}
\end{array}\right]
$$

with the generic matrix $\boldsymbol{A}$ satisfying the conditions

$$
\begin{aligned}
& a_{11}^{2}+a_{12}^{2}+a_{13}^{2}=\sigma_{Q}^{2}, \\
& a_{21}^{2}+a_{22}^{2}+a_{23}^{2}=\sigma_{Y_{1}}^{2}, \\
& a_{31}^{2}+a_{32}^{2}+a_{33}^{2}=\sigma_{Y_{2}}^{2},
\end{aligned}
$$




$$
\begin{aligned}
& a_{11} a_{21}+a_{12} a_{22}+a_{13} a_{23}=0, \\
& a_{11} a_{31}+a_{12} a_{32}+a_{13} a_{33}=\sigma_{Q} \sigma_{Y_{2}} \rho_{2}, \\
& a_{21} a_{31}+a_{22} a_{32}+a_{23} a_{33}=\sigma_{Y_{1}} \sigma_{Y_{2}} \rho,
\end{aligned}
$$

where $a_{i j}$ represents the element in the $i$ th line and $j$ th column of matrix $\boldsymbol{A}$.

It is immediate to conclude that the matrix $\boldsymbol{A}$ in (4) satisfies the conditions (29), as:

$$
\begin{aligned}
\sigma_{Q}^{2}\left(1-\frac{\rho_{2}^{2}}{1-\rho^{2}}\right)+\frac{\sigma_{Q}^{2} \rho_{2}^{2}}{1-\rho^{2}} & =\sigma_{Q}^{2}, \\
\sigma_{Y_{1}}^{2} & =\sigma_{Y_{1}}^{2}, \\
\sigma_{Y_{2}}^{2} \rho^{2}+\sigma_{Y_{2}}^{2}\left(1-\rho^{2}\right) & =\sigma_{Y_{2}}^{2}, \\
0 & =0, \\
\frac{\sigma_{Q} \rho_{2}}{\sqrt{1-\rho^{2}}} \sigma_{Y_{2}} \sqrt{1-\rho^{2}} & =\sigma_{Q} \sigma_{Y_{2}} \rho_{2}, \\
\sigma_{Y_{1}} \sigma_{Y_{2}} \rho & =\sigma_{Y_{1}} \sigma_{Y_{2}} \rho .
\end{aligned}
$$

\subsection{Expressions (13)-(16)}

\subsubsection{Optimal contamination drift (13)}

To obtain the equilibrium contamination drift vector $\boldsymbol{h}^{*}=\left[\begin{array}{lll}0 & 0 & h_{2}\end{array}\right]^{\top}$, we make use of Proposition 1 in Gagliardini et al. (2009), which implies that:

$$
h_{2}=-\sqrt{2 \eta} \frac{\sigma_{Y_{2}} \sqrt{1-\rho^{2}} V_{Y_{2}}+\frac{\sigma_{Q} \rho_{2}}{\sqrt{1-\rho^{2}}}}{\left.\sqrt{\left(\sigma_{Y_{2}} \sqrt{1-\rho^{2}}\right.} V_{Y_{2}}+\frac{\sigma_{Q} \rho_{2}}{\sqrt{1-\rho^{2}}}\right)^{2}},
$$

from which it is immediate to obtain (13).

\subsubsection{Equilibrium price of uncertainty (14)-(15)}

From Corollary 1 in Gagliardini et al. (2009), the equilibrium market premium of risk and ambiguity $(\boldsymbol{M})$ associated with the state variables $Y_{1 t}$ and $Y_{2 t}$ and the production process $Q_{t}$ is given by:

$$
\boldsymbol{M}=\boldsymbol{\sigma}^{\top}-\boldsymbol{h}^{*},
$$


with $\boldsymbol{\sigma}$ given by (5) and the equilibrium contamination drift vector $\boldsymbol{h}^{*}=\left[\begin{array}{lll}0 & 0 & h_{2}\end{array}\right]^{\top}$ given by (13). It is immediate to conclude that $\boldsymbol{M}$ is given by:

$$
\boldsymbol{M}=\left[\begin{array}{c}
\sigma_{Q} \sqrt{1-\frac{\rho_{2}^{2}}{1-\rho^{2}}} \\
0 \\
\frac{\sigma_{Q} \rho_{2}}{\sqrt{1-\rho^{2}}} \pm \sqrt{2 \eta}
\end{array}\right] .
$$

Following expression (18) in Gagliardini et al. (2009), the equilibrium market prices of uncertainty $\phi_{1}$ and $\phi_{2}$ associated with $Y_{1 t}$ and $Y_{2 t}$, respectively, are given by:

$$
\begin{aligned}
& \boldsymbol{\phi}=\left[\begin{array}{l}
\phi_{1} \\
\phi_{2}
\end{array}\right]=\boldsymbol{\Xi} \boldsymbol{M}=\left[\begin{array}{ccc}
0 & \sigma_{Y_{1}} & 0 \\
0 & \sigma_{Y_{2}} \rho & \sigma_{Y_{2}} \sqrt{1-\rho^{2}}
\end{array}\right]\left[\begin{array}{c}
\sigma_{Q} \sqrt{1-\frac{\rho_{2}^{2}}{1-\rho^{2}}} \\
0 \\
\frac{\sigma_{Q} \rho_{2}}{\sqrt{1-\rho^{2}}} \pm \sqrt{2 \eta}
\end{array}\right] \\
& \Leftrightarrow\left[\begin{array}{l}
\phi_{1} \\
\phi_{2}
\end{array}\right]=\left[\begin{array}{c}
0 \\
\sigma_{Q} \sigma_{Y_{2}} \rho_{2} \pm \sigma_{Y_{2}} \sqrt{2 \eta} \sqrt{1-\rho^{2}}
\end{array}\right],
\end{aligned}
$$

which, from (30), can be written as:

$$
\begin{aligned}
& \phi_{1}=0 \\
& \phi_{2}= \begin{cases}\sigma_{Y_{2}} \sigma_{Q} \rho_{2}+\sigma_{Y_{2}} \sqrt{2 \eta} \sqrt{1-\rho^{2}}, & \text { if } \sigma_{Y_{2}} \sqrt{1-\rho^{2}} V_{Y_{2}}+\frac{\sigma_{Q} \rho_{2}}{\sqrt{1-\rho^{2}}}>0, \\
\sigma_{Y_{2}} \sigma_{Q} \rho_{2}-\sigma_{Y_{2}} \sqrt{2 \eta} \sqrt{1-\rho^{2}}, & \text { if } \sigma_{Y_{2}} \sqrt{1-\rho^{2}} V_{Y_{2}}+\frac{\sigma_{Q} \rho_{2}}{\sqrt{1-\rho^{2}}}<0,\end{cases}
\end{aligned}
$$

which is (14) and (15), respectively.

\subsubsection{Equilibrium interest rate (16)}

From Corollary 1 in Gagliardini et al. (2009), the equilibrium instantaneous interest rate $r_{t}$ is given by:

$$
r_{t}=g_{Q}-\boldsymbol{\sigma} \boldsymbol{M}
$$

where $g_{Q}$ is the drift function in (1) and the matrices $\boldsymbol{\sigma}$ and $\boldsymbol{M}$ are given in (5) and (31), respectively. Consequently, $r_{t}$ is given by:

$$
\begin{gathered}
r_{t}=g_{Q}-\left[\begin{array}{lll}
\sigma_{Q} \sqrt{1-\frac{\rho_{2}^{2}}{1-\rho^{2}}} & 0 & \frac{\sigma_{Q} \rho_{2}}{\sqrt{1-\rho^{2}}}
\end{array}\right]\left[\begin{array}{c}
\sigma_{Q} \sqrt{1-\frac{\rho_{2}^{2}}{1-\rho^{2}}} \\
0 \\
\frac{\sigma_{Q} \rho_{2}}{\sqrt{1-\rho^{2}}} \pm \sqrt{2 \eta}
\end{array}\right] \\
\Leftrightarrow r_{t}=g_{Q}-\sigma_{Q}^{2} \pm \sqrt{2 \eta} \frac{\sigma_{Q} \rho_{2}}{\sqrt{1-\rho^{2}}},
\end{gathered}
$$


which from the previous Sect. 5.2.2, can be written as:

$$
r_{t}= \begin{cases}g_{Q}-\sigma_{Q}^{2}-\sqrt{2 \eta} \frac{\sigma_{Q} \rho_{2}}{\sqrt{1-\rho^{2}}}, & \text { if } \sigma_{Y_{2}} \sqrt{1-\rho^{2}} V_{Y_{2}}+\frac{\sigma_{Q} \rho_{2}}{\sqrt{1-\rho^{2}}}>0, \\ g_{Q}-\sigma_{Q}^{2}+\sqrt{2 \eta} \frac{\sigma_{Q} \rho_{2}}{\sqrt{1-\rho^{2}}}, & \text { if } \sigma_{Y_{2}} \sqrt{1-\rho^{2}} V_{Y_{2}}+\frac{\sigma_{Q} \rho_{2}}{\sqrt{1-\rho^{2}}}<0\end{cases}
$$

which is (16).

\subsection{Longstaff and Schwartz (1992): a particular case}

The model of Longstaff and Schwartz (1992) can be obtained as a particular case of our setting. Start by assuming:

$$
\begin{array}{r}
g_{Q}=\left(z Y_{1 t}+u Y_{2 t}\right) \text { and } \sigma_{Q}=v \sqrt{Y_{2 t}}, \\
g_{Y_{1}}=\left(a-b Y_{1 t}\right) \text { and } \sigma_{Y_{1}}=c \sqrt{Y_{1 t}}, \\
g_{Y_{2}}=\left(d-e Y_{2 t}\right) \text { and } \sigma_{Y_{2}}=f \sqrt{Y_{2 t}},
\end{array}
$$

where $z, u, v, a, b, c, d, e$ and $f$ are positive parameters. that:

Make the following change of variables: $y_{1 t}=\frac{Y_{1 t}}{c^{2}}$ and $y_{2 t}=\frac{Y_{2 t}}{f^{2}}$. This implies

$$
\begin{gathered}
g_{y_{1}}=\left(\gamma-\vartheta y_{1 t}\right) \text { and } \sigma_{y_{1}}=\sqrt{y_{1 t}}, \\
g_{y_{2}}=\left(\zeta-\xi y_{2 t}\right) \text { and } \sigma_{y_{2}}=\sqrt{y_{2 t}},
\end{gathered}
$$

where $\gamma=\frac{a}{c^{2}}, \vartheta=b, \zeta=\frac{d}{f^{2}}$ and $\xi=e$.

In the model of Longstaff and Schwartz (1992), the state variable $Y_{1 t}$ has uncorrelated shocks with those of economy's output rate and of the other state variable $Y_{2 t}$, i.e., $\rho=0$. Thus, matrix A in (4) becomes:

$$
\left[\begin{array}{ccc}
\sigma_{Q \sqrt{1-\rho_{2}^{2}}} & 0 & \sigma_{Q} \rho_{2} \\
0 & \sigma_{Y_{1}} & 0 \\
0 & 0 & \sigma_{Y_{2}}
\end{array}\right]
$$

Considering those specifications and the assumption in Longstaff and Schwartz (1992) that $\Lambda\left(W_{t}, Y_{1 t}, Y_{2 t}, t\right)=0$, from (17) one obtains the fundamental PDE satisfied by the contingent claim price $H\left(y_{1 t}, y_{2 t}, \tau\right)$ :

$$
\begin{aligned}
\frac{\partial H}{\partial \tau}= & \frac{1}{2}\left(\sqrt{y_{1 t}}\right)^{2} \frac{\partial^{2} H}{\partial y_{1}^{2}}+\frac{1}{2}\left(\sqrt{y_{2 t}}\right)^{2} \frac{\partial^{2} H}{\partial y_{2}^{2}}+\left(\gamma-\vartheta y_{1 t}\right) \frac{\partial H}{\partial y_{1}} \\
& +\left[\left(\zeta-\xi y_{2 t}\right)-\sqrt{y_{2 t}} \nu f \sqrt{y_{2 t}} \rho_{2}\right] \frac{\partial H}{\partial y_{2}}-r_{t} H \\
\Leftrightarrow & \frac{\partial H}{\partial \tau}=\frac{y_{1 t}}{2} \frac{\partial^{2} H}{\partial y_{1}^{2}}+\frac{y_{2 t}}{2} \frac{\partial^{2} H}{\partial y_{2}^{2}}+\left(\gamma-\vartheta y_{1 t}\right) \frac{\partial H}{\partial y_{1}}
\end{aligned}
$$




$$
+\left[\left(\zeta-\xi y_{2 t}\right)-\lambda y_{2 t}\right] \frac{\partial H}{\partial y_{2}}-r_{t} H,
$$

with $\lambda=\rho_{2} f v$, which is the PDE obtained by Longstaff and Schwartz (1992) [their Eqs. (8) and (9)]. ${ }^{12}$

Regarding the equilibrium interest rate, from (16) and the above specifications:

$$
r_{t}=g_{Q}-\sigma_{Q}^{2}=z c^{2} y_{1 t}+f^{2}\left(u-v^{2}\right) y_{2 t},
$$

which is the expression reached by Longstaff and Schwartz (1992) (their Eq. (10)).

\subsection{Concrete investment opportunity set}

\subsubsection{Bellman equation (24)}

Under the investment opportunity set (19), (20) and (23):

$$
\begin{aligned}
& \boldsymbol{g}_{\boldsymbol{Y}}=\left[\begin{array}{c}
\mu Y_{1 t} \\
\kappa\left(\theta-Y_{2 t}\right)
\end{array}\right] \\
& \boldsymbol{\Xi}=\left[\begin{array}{ccc}
0 & Y_{1 t} \sqrt{Y_{2 t}} & 0 \\
0 & \epsilon \sqrt{Y_{2 t}} \rho & \epsilon \sqrt{Y_{2 t}} \sqrt{1-\rho^{2}}
\end{array}\right] ; \\
& \boldsymbol{\sigma}^{\top}=\left[\begin{array}{c}
l \sqrt{Y_{2 t}} \sqrt{1-\frac{\rho_{2}^{2}}{1-\rho^{2}}} \\
0 \\
\frac{l \sqrt{Y_{2 t}} \rho_{2}}{\sqrt{1-\rho^{2}}}
\end{array}\right] \text {. }
\end{aligned}
$$

It is immediate to obtain:

$$
\begin{aligned}
& \boldsymbol{V}_{\boldsymbol{Y}}^{\top} \boldsymbol{g}_{\boldsymbol{Y}}=\left[\begin{array}{ll}
V_{Y_{1}} & V_{Y_{2}}
\end{array}\right]\left[\begin{array}{c}
\mu Y_{1 t} \\
\kappa\left(\theta-Y_{2 t}\right)
\end{array}\right]=\mu V_{Y_{1}} Y_{1 t}+\kappa\left(\theta-Y_{2 t}\right) V_{Y_{2}} ; \\
& \operatorname{trace}\left[\boldsymbol{\Xi}^{\top} \boldsymbol{V}_{\boldsymbol{Y} \boldsymbol{Y}} \boldsymbol{\Xi}\right]=Y_{1 t}^{2} Y_{2 t} V_{Y_{1} Y_{1}}+2 \epsilon \rho V_{Y_{2} Y_{1}} Y_{1 t} Y_{2 t}+\epsilon^{2} Y_{2 t} V_{Y_{2} Y_{2}} ; \\
& \boldsymbol{\Xi}^{\top} \boldsymbol{V}_{\boldsymbol{Y}}+\boldsymbol{\sigma}^{\top}=\left[\begin{array}{c}
l \sqrt{Y_{2 t}} \sqrt{1-\frac{\rho_{2}^{2}}{1-\rho^{2}}} \\
V_{Y_{1}} Y_{1 t} \sqrt{Y_{2 t}}+\epsilon \sqrt{Y_{2 t}} \rho V_{Y_{2}} \\
\epsilon \sqrt{Y_{2 t}} \sqrt{1-\rho^{2}} V_{Y_{2}}+\frac{l \sqrt{Y_{2 t} \rho_{2}}}{\sqrt{1-\rho^{2}}}
\end{array}\right] \\
& \left(\boldsymbol{\Xi}^{\top} \boldsymbol{V}_{\boldsymbol{Y}}+\boldsymbol{\sigma}^{\top}\right)^{\top}\left(\boldsymbol{\Xi}^{\top} \boldsymbol{V}_{\boldsymbol{Y}}+\boldsymbol{\sigma}^{\top}\right)=l^{2} Y_{2 t}\left(1-\frac{\rho_{2}^{2}}{1-\rho^{2}}\right)+\left(V_{Y_{1}} Y_{1 t} \sqrt{Y_{2 t}}\right.
\end{aligned}
$$

\footnotetext{
12 Note that the equilibrium price of risk associated with the "original" state variable $Y_{2 t}$ is also linear on its instantaneous level with the same coefficient $\lambda$, as $\sigma_{Q} \sigma_{Y_{2}} \rho_{2}=v \sqrt{Y_{2 t}} f \sqrt{Y_{2 t}} \rho_{2}=\rho_{2} v f Y_{2 t}=\lambda Y_{2 t}$, with $\lambda=\rho_{2} f v$.
} 


$$
\left.+\epsilon \sqrt{Y_{2 t}} \rho V_{Y_{2}}\right)^{2}+\left(\epsilon \sqrt{Y_{2 t}} \sqrt{1-\rho^{2}} V_{Y_{2}}+\frac{l \sqrt{Y_{2 t}} \rho_{2}}{\sqrt{1-\rho^{2}}}\right)^{2}=F\left(Y_{1 t}, Y_{2 t}\right) .
$$

Going back to (12), and substituting the obtained expressions:

$$
\begin{aligned}
\delta V= & \mu V_{Y_{1}} Y_{1 t}+\kappa\left(\theta-Y_{2 t}\right) V_{Y_{2}}+\ln Y_{1 t}+\alpha Y_{2 t}-\frac{1}{2} l^{2} Y_{2 t} \\
& +\frac{1}{2}\left(Y_{1 t}^{2} Y_{2 t} V_{Y_{1} Y_{1}}+2 \epsilon \rho V_{Y_{2} Y_{1}} Y_{1 t} Y_{2 t}+\epsilon^{2} Y_{2 t} V_{Y_{2} Y_{2}}\right) \\
& -\sqrt{2 \eta F\left(Y_{1 t}, Y_{2 t}\right)},
\end{aligned}
$$

which is (24).

\subsubsection{Value function (25)}

Start by noting that the value function $V\left(Y_{1 t}, Y_{2 t}\right)$ is a function of the two state variables, $Y_{1 t}$ and $Y_{2 t}$, for a given ambiguity parameter $\eta$, and therefore can be denoted as $V\left(Y_{1 t}, Y_{2 t}, \eta\right)$

$$
V: \mathbb{R}_{+} \times \mathbb{R}_{+} \times \mathbb{R}_{+} \longrightarrow \mathbb{R}
$$

When there is no ambiguity $(\eta=0)$, the Bellman equation (24) becomes:

$$
\begin{aligned}
\delta V= & \mu V_{Y_{1}} Y_{1 t}+\kappa\left(\theta-Y_{2 t}\right) V_{Y_{2}}+\frac{1}{2}\left(Y_{1 t}^{2} Y_{2 t} V_{Y_{1} Y_{1}}+2 \epsilon \rho V_{Y_{2} Y_{1}} Y_{1 t} Y_{2 t}+\epsilon^{2} Y_{2 t} V_{Y_{2} Y_{2}}\right) \\
& +\ln Y_{1 t}+\alpha Y_{2 t}-\frac{1}{2} l^{2} Y_{2 t}
\end{aligned}
$$

which is solved by the value function:

$$
V\left(Y_{1 t}, Y_{2 t}, \eta=0\right)=a \ln Y_{1 t}+b Y_{2 t}+c,
$$

where

$$
a=\frac{1}{\delta}, \quad b=\frac{\left(\alpha-\frac{l^{2}}{2}-\frac{1}{2 \delta}\right)}{(\kappa+\delta)} \text { and } c=\frac{\mu}{\delta^{2}}+\frac{\kappa \theta}{\delta} b .
$$

Proof From (33)

$$
V_{Y_{1}}=\frac{a}{Y_{1 t}}, \quad V_{Y_{1} Y_{1}}=-\frac{a}{Y_{1 t}^{2}}, \quad V_{Y_{1} Y_{2}}=0, \quad V_{Y_{2}}=b, \quad V_{Y_{2} Y_{2}}=0 .
$$

Considering these results, (33) and (34), and plugging them into (32) one gets:

$$
\delta\left(\frac{1}{\delta} \ln Y_{1 t}+b Y_{2 t}+c\right)=\mu \frac{a}{Y_{1 t}} Y_{1 t}+\kappa\left(\theta-Y_{2 t}\right) b+\frac{1}{2}\left(-Y_{1 t}^{2} Y_{2 t} \frac{a}{Y_{1 t}^{2}}\right)+\ln Y_{1 t}
$$




$$
\begin{aligned}
&+\alpha Y_{2 t}-\frac{l^{2}}{2} Y_{2 t} \\
& \Leftrightarrow \ln Y_{1 t}+\delta b Y_{2 t}+\delta c=\mu a+\kappa\left(\theta-Y_{2 t}\right) b-\frac{1}{2} Y_{2 t} a+\ln Y_{1 t}+\alpha Y_{2 t}-\frac{l^{2}}{2} Y_{2 t} \\
& \Leftrightarrow \delta c-\frac{\mu}{\delta}-b \kappa \theta= {\left[\alpha-\frac{l^{2}}{2}-\frac{1}{2 \delta}-b(\kappa+\delta)\right] Y_{2 t} } \\
& \Leftrightarrow \frac{\mu}{\delta}+\kappa \theta b-\frac{\mu}{\delta}-b \kappa \theta= {\left[\alpha-\frac{l^{2}}{2}-\frac{1}{2 \delta}-\frac{\left(\alpha-\frac{l^{2}}{2}-\frac{1}{2 \delta}\right)}{(\kappa+\delta)}(\kappa+\delta)\right] Y_{2 t} } \\
& \Leftrightarrow 0= 0,
\end{aligned}
$$

as we wanted to prove.

From previous assumptions that $V_{Y_{2}}$ and $\frac{\partial V_{Y_{2}}}{\partial \eta}$ exist in the domain $0<\eta<\Psi$ (with $\Psi$ being a small positive number), results that in this domain for $\eta, V\left(Y_{1 t}, Y_{2 t}, \eta>0\right) \approx$ $V\left(Y_{1 t}, Y_{2 t}, \eta=0\right)$, implying $\left.\left.V_{Y_{2}}\right|_{\eta>0} \approx V_{Y_{2}}\right|_{\eta=0}=b$, where $b$ is given by (34).

\subsubsection{Equilibrium uncertainty price (26) and interest rate (28)}

From (15) and (16), a key issue is the sign of $V_{Y_{2}} \sigma_{Y_{2}}\left(1-\rho^{2}\right)+\sigma_{Q} \rho_{2}$. Under the concrete investment opportunity set:

$$
V_{Y_{2}} \sigma_{Y_{2}}\left(1-\rho^{2}\right)+\sigma_{Q} \rho_{2}=\left[\frac{\epsilon\left(1-\rho^{2}\right)}{(\kappa+\delta)}\left(\alpha-\frac{1}{2} l^{2}-\frac{1}{2 \delta}\right)+l \rho_{2}\right] \sqrt{Y_{2 t}}
$$

implying that the sign of $V_{Y_{2}} \sigma_{Y_{2}}\left(1-\rho^{2}\right)+\sigma_{Q} \rho_{2}$ is the sign of $\left[\frac{\epsilon\left(1-\rho^{2}\right)}{(\kappa+\delta)}\left(\alpha-\frac{1}{2} l^{2}-\frac{1}{2 \delta}\right)\right.$ $\left.+l \rho_{2}\right]$. The threshold value $\omega$ in (26) and (28) is therefore obtained:

$$
\begin{aligned}
0 & =\frac{\epsilon\left(1-\rho^{2}\right)}{(\kappa+\delta)}\left(\alpha-\frac{1}{2} l^{2}-\frac{1}{2 \delta}\right)+l \rho_{2} \\
\Leftrightarrow \alpha= & \underbrace{\frac{\left(l^{2} \delta \epsilon+\epsilon\right)\left(1-\rho^{2}\right)-2 \delta(\kappa+\delta) l \rho_{2}}{2 \delta \epsilon\left(1-\rho^{2}\right)}}_{\omega} .
\end{aligned}
$$

When $\alpha>\omega($ resp. $\alpha<\omega)$, then $V_{Y_{2}} \sigma_{Y_{2}}\left(1-\rho^{2}\right)+\sigma_{Q} \rho_{2}>0($ resp. $<0)$, making it immediate to obtain (26) from (15) and (28) from (16).

\section{References}

Amin, K., Ng, V.: Option valuation with systematic stochastic volatility. J Financ 48, 881-910 (1993)

Barillas, F., Hansen, L.P., Sargent, T.J.: Doubts or variability? J Econ Theory 144, 2388-2418 (2009)

Black, F., Scholes, M.S.: The pricing of options and corporate liabilities J Polit Econ 81, 637-654 (1973) 
Brennan, M.J., Schwartz, E.S.: A continuous time approach to the pricing of bonds J Bank Financ 3, 133155 (1979)

Cagetti, M., Hansen, L.P., Sargent, T.J., Williams, N.: Robustness and pricing with uncertain growth Rev Financ Stud 15, 363-404 (2002)

Camerer, C., Weber, M.: Recent developments in modeling preferences: uncertainty and ambiguity J Risk Uncertain 5, 325-370 (1992)

Cao, H.H., Wang, T., Zhang, H.H.: Model uncertainty, limited market participation, and asset prices Rev Financ Stud 18, 1219-1251 (2005)

Chen, Z., Epstein, L.G.: Ambiguity, risk, and asset returns in continuous time Econometrica 70, 14031443 (2002)

Correia-da-Silva, J., Hervés-Beloso, C.: Prudent expectations equilibrium in economies with uncertain delivery Econ Theory 39, 67-92 (2009)

Cox, J.C., Ingersoll, J.E., Ross, S.A.: An intertemporal general equilibrium model of asset prices Econometrica 53, 363-384 (1985)

Cox, J.C., Ingersoll, J.E., Ross, S.A.: A theory of the term structure of interest rates Econometrica 53, 385407 (1985)

de Castro, L., Pesce, M., Yannelis, N.: Core and equilibria under ambiguity Econ Theory 48, 519-548 (2011)

Dow, J., Werlang, S.R.: Uncertainty aversion, risk aversion, and the optimal choice of portfolio Econometrica 60, 197-204 (1992)

Duffie, D., Epstein, L.G.: Asset pricing with stochastic differential utility Rev Financ Stud 5, 411-436 (1992)

Duffie, D., Epstein, L.G.: Stochastic differential utility Econometrica 60, 353-394 (1992)

Eichberger, J., Kelsey, D.: Are the treasures of game theory ambiguous? Econ Theory 48, 313-339 (2011)

Ellsberg, D.: Risk, ambiguity, and the Savage axioms Q J Econ 75, 643-669 (1961)

Epstein, L.G.: A paradox for the smooth ambiguity model of preference Econometrica 78, 2085-2099 (2010)

Epstein, L.G., Miao, J.: A two-person dynamic equilibrium under ambiguity J Econ Dyn Control 27, 12531288 (2003)

Epstein, L.G., Schneider, M.: Recursive multiple-priors J Econ Theory 113, 1-31 (2003)

Epstein, L.G., Schneider, M.: Ambiguity and asset markets Annu Rev Financ Econ 2, 315-346 (2010)

Epstein, L.G., Wang, T.: Intertemporal asset pricing under knightian uncertainty Econometrica 62, 283322 (1994)

Eraker, B., Shaliastovich, I.: An equilibrium guide to designing affine pricing models Math Financ 18, 519543 (2008)

Etner, J., Jeleva, M., Tallon, J.M.: Decision theory under ambiguity J Econ Surv 26, 234-270 (2012)

Faria, G., Correia-da-Silva, J.: Closed-form solution for options with ambiguity about stochastic volatility. RGEA working papers 6-11, Universidade de Vigo, Research Group in Economic Analysis (2011)

Gagliardini, P., Porchia, P., Trojani, F.: Ambiguity aversion and the term structure of interest rates Rev Financ Stud 22, 4157-4188 (2009)

Garlappi, L., Uppal, R., Wang, T.: Portfolio selection with parameter and model uncertainty: a multi-prior approach Rev Financ Stud 20, 41-81 (2007)

Gilboa, I., Schmeidler, D.: Maxmin expected utility with non-unique prior J Math Econ 18, 141-153 (1989)

Hansen, L.P., Sargent, T.J.: Acknowledging misspecification in macroeconomic theory Rev Econ Dyn 4, 519-535 (2001)

Hansen, L.P., Sargent, T.J.: Time inconsistency of robust control? Unpublished manuscript (2006)

Hansen, L.P., Sargent, T.J., Tallarini, T.D.: Robust permanent income and pricing Rev Econ Stud 66, 873907 (1999)

Hansen, L.P., Sargent, T.J., Wang, N.E.: Robust permanent income and pricing with filtering Macroecon Dyn 6, 40-84 (2002)

Heston, S.L.: A closed-form solution for options with stochastic volatility with applications to bond and currency options Rev Financ Stud 6, 327-343 (1993)

Ilut, C., Schneider, M.: Ambiguous business cycles. NBER working paper 17900 (2012)

Klibanoff, P., Marinacci, M., Mukerji, S.: A smooth model of decision making under ambiguity Econometrica 73, 1849-1892 (2005)

Klibanoff, P., Marinacci, M., Mukerji, S.: On the smooth ambiguity model: a reply. Econometrica (2011, forthcoming)

Knight, F.H.: Risk, Uncertainty and Profit. Houghton Mifflin, Boston (1921)

Leippold, M., Trojani, F., Vanini, P.: Learning and asset prices under ambiguous information Rev Financ Stud 21, 2565-2597 (2008) 
Liu, H.: Robust consumption and portfolio choice for time varying investment opportunities Ann Financ 6, 435-454 (2010)

Longstaff, F.A., Schwartz, E.S.: Interest rate volatility and the term structure: a two-factor general equilibrium model J Financ 47, 1259-1282 (1992)

Lucas, R.: Asset prices in an exchange economy Econometrica 46, 1429-1445 (1978)

Ma, X., Zhao, Q., Qu, J.: Robust portfolio optimization with a generalized expected utility model under ambiguity Ann Financ 4, 431-444 (2008)

Maccheroni, F., Marinacci, M., Rustichini, A.: Ambiguity aversion, robustness, and the variational representation of preferences Econometrica 74, 1447-1498 (2006)

Maenhout, P.J.: Robust portfolio rules and asset pricing Rev Financ Stud 17, 951-983 (2004)

Maenhout, P.J.: Robust portfolio rules and detection-error probabilities for a mean-reverting risk premium J Econ Theory 128, 136-163 (2006)

Ozsoylev, H., Werner, J.: Liquidity and asset prices in rational expectations equilibrium with ambiguous information Econ Theory 48, 469-491 (2011)

Riedel, F.: Optimal stopping with multiple priors Econometrica 77, 857-908 (2009)

Trojani, F., Vanini, P.: Robustness and ambiguity aversion in general equilibrium Rev Financ 8, 279324 (2004)

Ui, T.: The ambiguity premium vs the risk premium under limited market participation Rev Financ 15, 245$275(2011)$

Ulrich, M.: Observable long-run ambiguity and long-run risk. Working paper, Columbia Business School (2010)

Uppal, R., Wang, T.: Model misspecification and underdiversification J Financ 58, 2465-2486 (2003)

Vasicek, O.: An equilibrium characterization of the term structure J Financ Econ 5, 177-188 (1977)

$\mathrm{Xu}, \mathrm{W} ., \mathrm{Li}, \mathrm{H} ., \mathrm{Wu}, \mathrm{C} .:$ A robust general equilibrium stochastic volatility model with recursive preference investors Ann Econ Financ 12, 217-231 (2011)

Zhang, W., Semmler, W.: Monetary policy rules under uncertainty: empirical evidence, adaptative learning, and robust control Macroecon Dyn 9, 651-681 (2005) 